\title{
Injection moulding of long glass fibre reinforced polyamide 6-6: guidelines to improve flexural properties
}

\author{
E. Lafranche ${ }^{*}$, P. Krawczak ${ }^{1}$, J. P. Ciolczyk ${ }^{2}$, J. Maugey ${ }^{2}$ \\ ${ }^{1}$ Ecole des Mines de Douai, Polymers and Composites Technology \& Mechanical Engineering Department, 941 rue \\ Charles Bourseul, BP 10838, 59508 Douai, France \\ ${ }^{2}$ Hutchinson SA, Research Department, Rue Gustave Nourry, BP31, 45120 Châlette-sur-Loing, France
}

Received 19 March 2007; accepted in revised form 3 June 2007

\begin{abstract}
Based on a previous optimisation of set-up parameters for injection moulding of polyamide 6-6 (PA 6-6) reinforced with $40 \mathrm{wt} \%$ of $10 \mathrm{~mm}$ long glass fibre, the aim of this paper is to define suitable guidelines to further improve the mechanical performances of PA 6-6/glass long fibre thermoplastic (LFT) injection mouldings. Different solutions have been considered: screw and non-return valve design modification so as to adapt them to LFT processing, increase of the initial fibre content (up to $50 \mathrm{wt} \%$ ) and length (up to $25 \mathrm{~mm}$ ) in the LFT pellets. Using a LFT dedicated plasticating unit has allowed decreasing the fibre breakage amount by about $80 \%$ at the nozzle exit, however without improving the flexural properties. Increasing the initial fibre content has logically permitted to improve the flexural properties. Increasing the initial fibre length has not brought any improvement of the properties and has even amplified the structural heterogeneities and anisotropy of the parts. These trends have been explained on the basis of cavity pressure records highlighting significantly different rheological behaviours, and of resulting residual fibre lengths and through-the-thickness fibre orientation profiles modifications.
\end{abstract}

Keywords: polymer composites, long fibre thermoplastics, fibre content distribution, fibre orientation, mechanical properties

\section{Introduction}

The ever-growing use of reinforced thermoplastics in various industrial applications has led to a demand of always-higher mechanical performances for injection-moulded parts. However because of the low residual fibre length after processing, mechanical properties are often limited when short fibre reinforced thermoplastic pellets are used. In order to overcome this limitation, long fibre thermoplastic (LFT) pellets have been developed so as to answer these new market requirements through a higher fibre aspect ratio (length/diameter ratio) theoretically leading to higher mechanical properties. The improvement of mechanical properties however also depends on the homogeneity and isotropy of the injection-moulded plastic parts, which are governed by the fibre distribution and orientation mechanisms, and on the capacity of the processing technologies to limit fibre breakage.

In the case of the injection moulding process, preservation of the reinforcement integrity begins at the pellet plastication stage. Yilmazer et al. [1] have shown that the screw rotation speed and the plastication volumetric flow rate during the feeding stage act according to two combined effects. First, the increase of the screw rotation speed induces higher shear stresses in the melt, which increases the fibre breakage. Then, this fibre breakage stabilizes due to the viscous dissipation that increases the melt temperature and thus decreases its viscos- 
ity. These results have confirmed the observations made for short fibre reinforced polyamides by Obieglo et al. [2]. Their work focused on the nonreturn valve and screw geometries has shown that short non-return valves warrant lower fibre breakage. On the opposite, other authors $[3,4]$ have recommended short plastication times (high screw rotation speeds, low back pressure) and pellet preheating before plastication in order to decrease fibre breakage.

Also the injection moulding conditions influence the fibre breakage because of the high shear stresses induced by the melt flowing through the gates or within the mould cavities. Especially, high injection speeds associated to low temperatures have a strong detrimental effect on reinforcement integrity [3-5]. The holding pressure level has an effect, which is all the more important than the injection speed is high. This is attributed to the fibre movements induced by a secondary flow within the melt core, whose viscosity quickly increases during the cooling stage of the part [3]. On the other side, a low level of holding pressure induces a poor interfacial adhesion of the matrix around the fibres and increases the parts void content $[5,6]$.

Moreover, the initial fibre fraction and aspect ratio (length/diameter) also influence the residual fibre length. Some authors [7-9] have shown that the fibre damage is higher when the fibre content or the fibre aspect ratio increase, that is to say when the fibre-fibre interactions are more important. High fibre contents also generate fibre clusters leading to local heterogeneity [9]. The resulting mechanical properties are greatly dependent on these factors $[3,8,10,11]$.

Besides, the velocity fields generated by the melt flow during the different stages of the injectionmoulding process induce a multi-layered composite structure within the part (so-called skin-core effect). Most of the injection-moulded parts have a low thickness. Therefore, fibres generally tend to orient in the plane of flow. If the fibre orientation mechanisms of long fibre reinforced thermoplastics are generally the same as those noticed for short fibre reinforced thermoplastics (e.g. existence of a fountain flow) [5], the core layer is thicker when the fibre length increases $[6,7,12]$. In addition, other studies have shown that whatever is the reinforcement length (short or long) the skin layers thickness remains roughly the same whereas the core layer thickness increases when the cavity thickness is higher $[13,14]$. This typical multi-layered structure also greatly influences the mechanical performances of the manufactured parts [10].

Finally, different authors have highlighted that the flow of the reinforced polymer melt in the mould cavity induces a concentration gradient of fibres along the flow axis, along the plate width and through the part thickness [5, 6, 15-17]. This segregation effect is noticed for both short and long fibre reinforced thermoplastics. The fibre segregation along the flow axis is however all the more important as the fibre length or the initial fibre fraction are high. This phenomenon is strongly amplified by the presence of any sharp geometrical discontinuity along the flow path $[5,6,17]$. The fibre accumulation (higher fibre contents) towards the end and in the centre (core) of the part have been attributed to the shear stresses at interfaces between the molten core and the frozen skin layers, which produce breakage of partially embedded fibres. The broken fibres are then pulled out and swept along by the melt flow and thus feed the next section of the part $[15,16]$. Kamal and Singh have also explained the fibre content decrease at the cavity edges by the peculiarities of the velocity flied at this location [18]. The homogeneity of the injection-moulded part also depend on the processing conditions, in particular the moulding temperatures [5].

A previous experimental investigation of injection moulding of polyamide 6-6 reinforced with $40 \mathrm{wt} \%$ of $10 \mathrm{~mm}$ long glass fibres had already allowed to identify the most influential processing parameters (mainly melt temperature and volume flow rate) and to optimise them so as to achieve flexural properties as high as possible [5]. The limits of the plasticating unit design (screw and non-return valve), which greatly influences the fibre breakage (and therefore the residual fibre length), had been however also pointed out. Consequently, there is a need to define further guidelines to possibly further improve the flexural property of polyamide 6-6/ glass LFT injection mouldings. The aim of this paper is to focus on the effect of the plasticating unit (different screw and non-return valve design) and of the LFT pellets type (various initial fibre lengths and weight fractions). The mechanical properties/microstructure (heterogeneity, anisotropy) relationship is going to be investigated on the basis of fibre content distributions, local 
residual fibre lengths and average fibre orientations measurements in the injection-moulded parts.

\section{Experimental}

\subsection{Materials}

The materials studied are polyamides 6-6 (PA 6-6) reinforced by $40 \mathrm{wt} \%$ or $50 \mathrm{wt} \%$ of $10 \mathrm{~mm}$ or $25 \mathrm{~mm}$ long glass fibres (Ticona). These materials will be referenced LGF $40 \mathrm{wt} \%$ - $10 \mathrm{~mm}$; LGF $50 \mathrm{wt} \%-10 \mathrm{~mm}$ and LGF $50 \mathrm{wt} \%-25 \mathrm{~mm}$.

\subsection{Machine and mould}

The experiments were carried out on a $2000 \mathrm{kN}$ clamping force injection-moulding machine (DK Codim). The machine has a vertical clamping unit (injection gate located in the mould parting line), and is equipped either with a standard all-purpose $55 \mathrm{~mm}$ diameter screw or with a $55 \mathrm{~mm}$ diameter screw specially designed for long glass fibre processing (Euro-Stel).

The prototype injection mould is a rectangular plate of $300 \times 120 \times 3 \mathrm{~mm}$. A $4 \mathrm{~mm}$ thick fan gate feeds the cavity over its whole width (unidirectional flow in the longitudinal direction of the plate). This mould was specially designed so as to reproduce some geometrical discontinuities (like sharp frontal and tangential steps) occurring on real industrial moulds that locally disturb the melt flow and consequently induce local microstructural heterogeneity (Figure 1). The mould temperature is controlled by means of independent channels, which ensures the surface temperature homogeneity with an accuracy of $\pm 1^{\circ} \mathrm{C}$. Three pressure transducers are

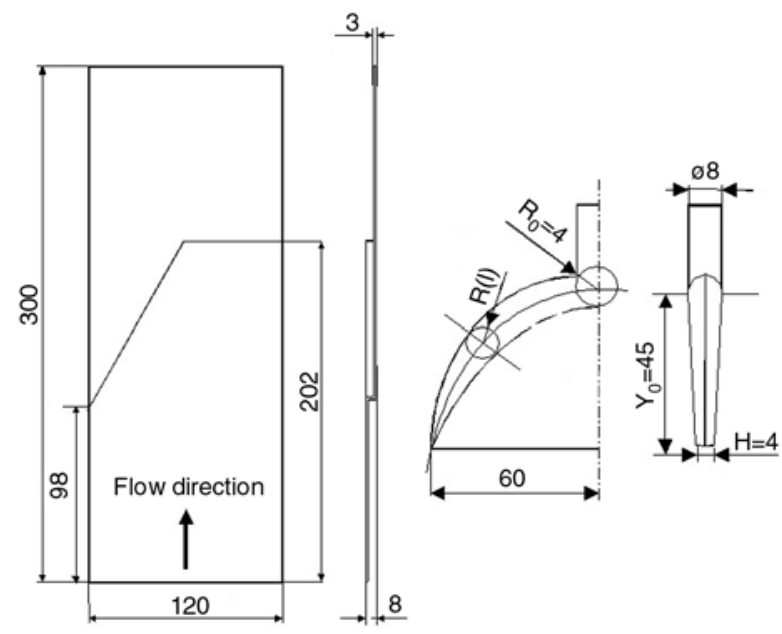

Figure 1. Plastic part and fan gate geometry
Table 1. Moulding conditions for LGF PA 6-6

\begin{tabular}{|l|c|}
\hline \multicolumn{1}{|c|}{ Processing parameters } & Set up value \\
\hline Mould temperature $\left[{ }^{\circ} \mathrm{C}\right]$ & 120 \\
\hline Melt temperature $\left[{ }^{\circ} \mathrm{C}\right]$ & 300 \\
\hline Volume flow rate $\left[\mathrm{cm}^{3} / \mathrm{s}\right]$ & 83 \\
\hline Holding pressure $[\mathrm{bar}]$ & 440 \\
\hline Back pressure $[\mathrm{bar}]$ & 8 \\
\hline Screw rotation speed $[\mathrm{rpm}]$ & 60 \\
\hline
\end{tabular}

located at $40 \mathrm{~mm}, 110 \mathrm{~mm}$ and $220 \mathrm{~mm}$ from the gate and are associated with two temperature sensors at $40 \mathrm{~mm}$ and $220 \mathrm{~mm}$.

\subsection{Moulding conditions}

The optimum moulding conditions previously determined [5] to achieve the highest flexural properties in case of $40 \mathrm{wt} \%$ of $10 \mathrm{~mm}$ long glass fibre reinforced PA 6-6 injection moulding are reported in Table 1.

\subsection{Mechanical testing}

Bending test leading to the determination of the flexural properties (strength, defined as maximum stress, and modulus) were performed according to ISO 178 on a standard tensile machine (Instron) at $2 \mathrm{~mm} / \mathrm{min}$, on five samples (dimensions $60 \times 25 \times$ $3 \mathrm{~mm}$ ) in both flow (longitudinal, 1) and transverse (2) directions before (beginning) and after (end) the geometrical discontinuities as shown in Figure 2.

\subsection{Structure analysis}

The fibre lengths, fibre contents (fibre weight fractions) and fibre orientations were measured at the
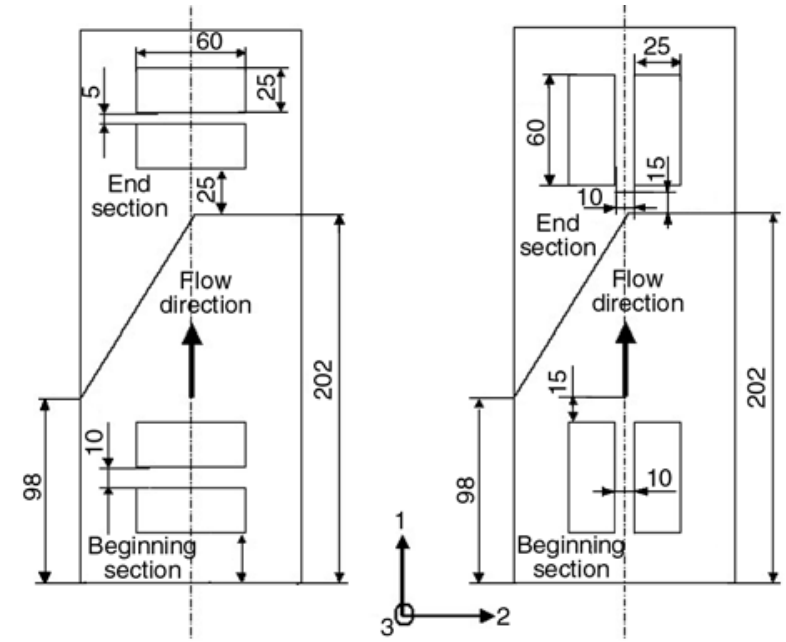

Figure 2. Location of test samples in the plate 
locations shown in Figure 3 as described in detail in the reference [5].

The local fibre content through the thickness was measured on $200 \mu \mathrm{m}$ thick slices peeled from a $12 \times 5 \mathrm{~mm}$ sample cut out of the $20 \times 20 \mathrm{~mm}$ coupons.

The fibre orientations were described using a second order orientation tensor as explained hereafter. Long fibres do not remain perfectly straight in the injection moulded parts. It was therefore required to find out a method to appreciate the average orientation of a tortuous curved fibre. However, no dedicated assessment protocol has been identified in the literature. As the observation of cross-sections through the part thickness has confirmed the planar orientation of the fibres, the curved long fibres have been divided in multiple adjacent straight segments oriented in the injection plane (Figure 4).

In that case each segment can be considered as a single straight fibre and its orientation can be easily described by two angles $\theta$ and $\phi$ (Figure 5), as for short fibres. The directions 1, 2, and 3 refer respectively to the flow, width and thickness directions. The orientation state of a sample can be described by a probability distribution function $\psi(\theta, \phi)$, which expresses the probability of finding a fibre within a given angular interval $[\phi, \phi+\mathrm{d} \phi]$ and $[\theta, \theta+\mathrm{d} \theta]$ at time $t$. An alternative to describe this orientation state is to associate the unit vector $\underline{p}$ to the fibre

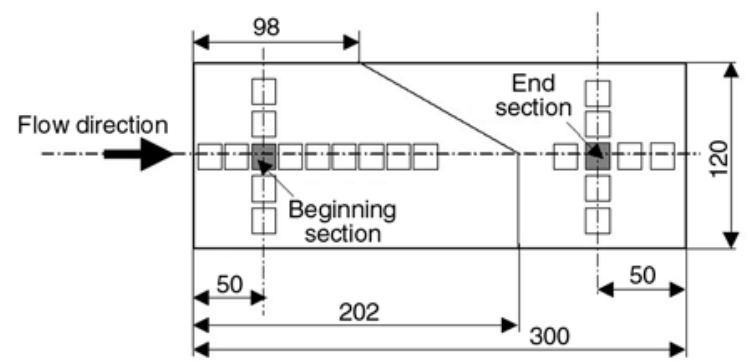

Figure 3. Cutting pattern of test samples for fibre content (white coupons) and fibre length and orientation (black coupons) determination [5]

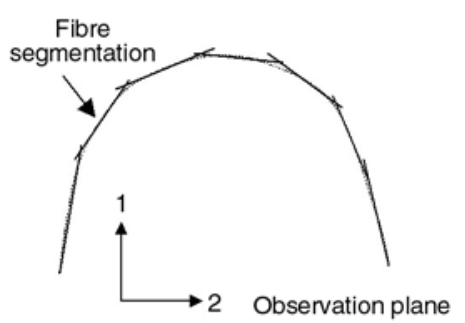

Figure 4. Curved fibre segmentation for orientation measurement

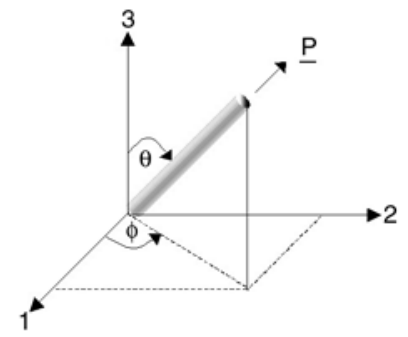

Figure 5. Description of fibre orientation angles

direction, as shown in Figure 5. The orientation distribution function can then be written as a function $\psi(p, t)$, which expresses the probability of finding a fibre with a given orientation $\underline{p}$ at time $t$. This distribution function has to satisfy some physical requirements. Since the fibre can rotate of $180^{\circ}$ around the axe 1 without changing the global orientation, $\psi(\underline{p}, t)=\psi(-\underline{p}, t)$.

Nevertheless, this orientation function is difficult to handle. Advani and Tucker [19] thus used the notion of orientation tensors, in order to obtain a complete description of the orientation state from a small number of discrete values. Such tensors are defined as the dyadic products of the unit vector $\underline{p}$ averaged over all possible directions, with $\psi$ as weighting function. Using an orientation tensor is equivalent to approximating the orientation distribution function by a finite term number in a Fourier series. This method has been chosen to describe the orientation state of the moulded parts. The second order tensor $\underset{=2}{a}$, defined by Equation (1), has been used.

$\underset{\underline{a}}{a}=a_{i j}=\oint p_{i} p_{j} \psi(\underline{p}, t) \delta \underline{p}$

These tensor values characterize the orientation state with respect to a reference direction (observation direction 1), usually the flow direction:

$a_{11}<0.30$ orientation perpendicular to flow direction (core layer)

$a_{11}=0.5$ perfectly random orientation

$a_{11}>0.7$ orientation parallel to flow direction

For the fibre orientation measurements, $20 \times 20 \mathrm{~mm}$ samples were cut (Figure 3 ) and subjected to successive stages of polishing through the thickness (with sandpaper of progressively smaller roughness) to obtain smooth surfaces. The images were obtained thanks to a light microscope (Jenapol, Carl Zeiss Jena), CCD camera and an image analysis software package (Visilog ${ }^{\circledR}$ 5.2, Noesis). The experimental procedure described by Bay and 
Tucker [12] was used to get the components of the orientation tensor.

\section{Results and discussion}

\subsection{Influence of screw and non-return valve design}

The fibre breakage during the plastication stage depends not only on the processing conditions (set up values of the screw rotation speed and back pressure) [1-5], but also on screw and non-return valve design. Actually, the fibre damage may occur in different manners:

- in the feed section of the screw (solid zone) directly within the pellets due to their solid-state compaction, which depends on the flight depth,

- in the compression section, where the coexistence of solid and molten polymer phases, coupled to the increasing compression ratio along this part of the screw, induces high shear rates,

- in the metering section, where the molten matrix imposes flexural stresses to the fibres,

- during flowing through the non-return valve due to the flow section reduction.

Previous studies $[4,5]$ have shown that the fibre breakage principally occurs in the compressive section of the screw. Therefore, raw material producers usually recommend a screw profile adapted to their LFT's materials [20]. The screw is designed so as to decrease the shear stresses within the barrel during the plasticating and injection stages (lower compression ratio, longer compression section, higher flight depth in feed section, shorter sliding ring...). However, due to evident economical considerations or just to keep a multi-material character of their injection-moulding machines, most of plastic parts manufacturers prefer to use their standard plasticating units to process LFT materials. It is therefore worth evaluating the benefit brought by a LGF (Long Glass Fibre) dedicated screw and non-return valve in terms of mechanical performances. The geometrical characteristics of such a LGF dedicated plasticating unit are reported in Table 2 compared to a standard one.

The LGF plasticating unit surprisingly induces a global decrease of flexural properties, except for the flexural strength in the transverse direction at the beginning of the plate (Table 3). The part appears to be more isotropic in its beginning section when the flow is not perturbed by the geomet-
Table 2. Characteristics of the LGF plasticating unit compared to those of the standard one

\begin{tabular}{|l|c|}
\hline & LGF/standard ratio \\
\hline Feed section length & 0.92 \\
\hline Compression section length & 1.76 \\
\hline Metering section length & 0.65 \\
\hline Flight depth in feed section & 1.23 \\
\hline Flight depth in metering section & 1.33 \\
\hline Compression ratio & 0.92 \\
\hline Flight pitch & 0.87 \\
\hline Sliding ring length & 0.87 \\
\hline
\end{tabular}

Table 3. Influence of plasticating unit type on flexural properties of LGF $40 \mathrm{wt} \%$ - $10 \mathrm{~mm}$ injectionmoulded parts

\begin{tabular}{|l|c|c|}
\hline \multirow{2}{*}{\multicolumn{1}{|c|}{ Property* $^{*}$}} & \multicolumn{2}{c|}{$\begin{array}{c}\text { Property variation } \\
\text { for LGF unit } \\
\text { (compared to standard unit) }\end{array}$} \\
\cline { 2 - 3 } & $\begin{array}{c}\text { Beginning } \\
\text { section }\end{array}$ & $\begin{array}{c}\text { End } \\
\text { section }\end{array}$ \\
\hline Longitudinal strength $\sigma_{1}[\mathrm{MPa}]$ & $\approx$ & $-10 \%$ \\
\hline Transverse strength $\sigma_{2}[\mathrm{MPa}]$ & $+7 \%$ & $-13 \%$ \\
\hline Longitudinal modulus $E_{1}[\mathrm{MPa}]$ & $-10 \%$ & $-15 \%$ \\
\hline Transverse modulus $E_{2}[\mathrm{MPa}]$ & $-17 \%$ & $-15 \%$ \\
\hline Anisotropy ratio $\sigma_{1} / \sigma_{2}$ & $-10 \%$ & $+4 \%$ \\
\hline
\end{tabular}

*Sample location according to Figure 2.

rical discontinuities. The presence of sharp longitudinal and frontal steps increases in that case the anisotropy ratio. Such properties evolutions are going to be related to microstructure modifications (e.g. fibre residual lengths, local fibre content and orientation distributions).

Also, the modification of the screw and non-return valve design seems to have induced thermal changes in the melt, which have consequently modified the rheological behaviour of the material during the injection cycle. Such a trend has also been recorded for PA6/glass LFTs [21] and may be attributed to the increase of the compression zone length, which leads to a larger polymer melt/barrel wall contact area. The indirect evidence of this effect also appears on the cavity pressure records. Sign of a better pressure transfer efficiency, an increase of pressure levels is noticed when the material is moulded with the LGF unit (Figure 6). Moreover Figure $6 \mathrm{~b}$ also shows a significant cooling rate decrease leading to longer cooling times. The ability of the LGF dedicated plasticating unit to maintain the reinforcement integrity during processing is clearly demonstrated. The fibre breakage amount is reduced in an extent reaching more than 

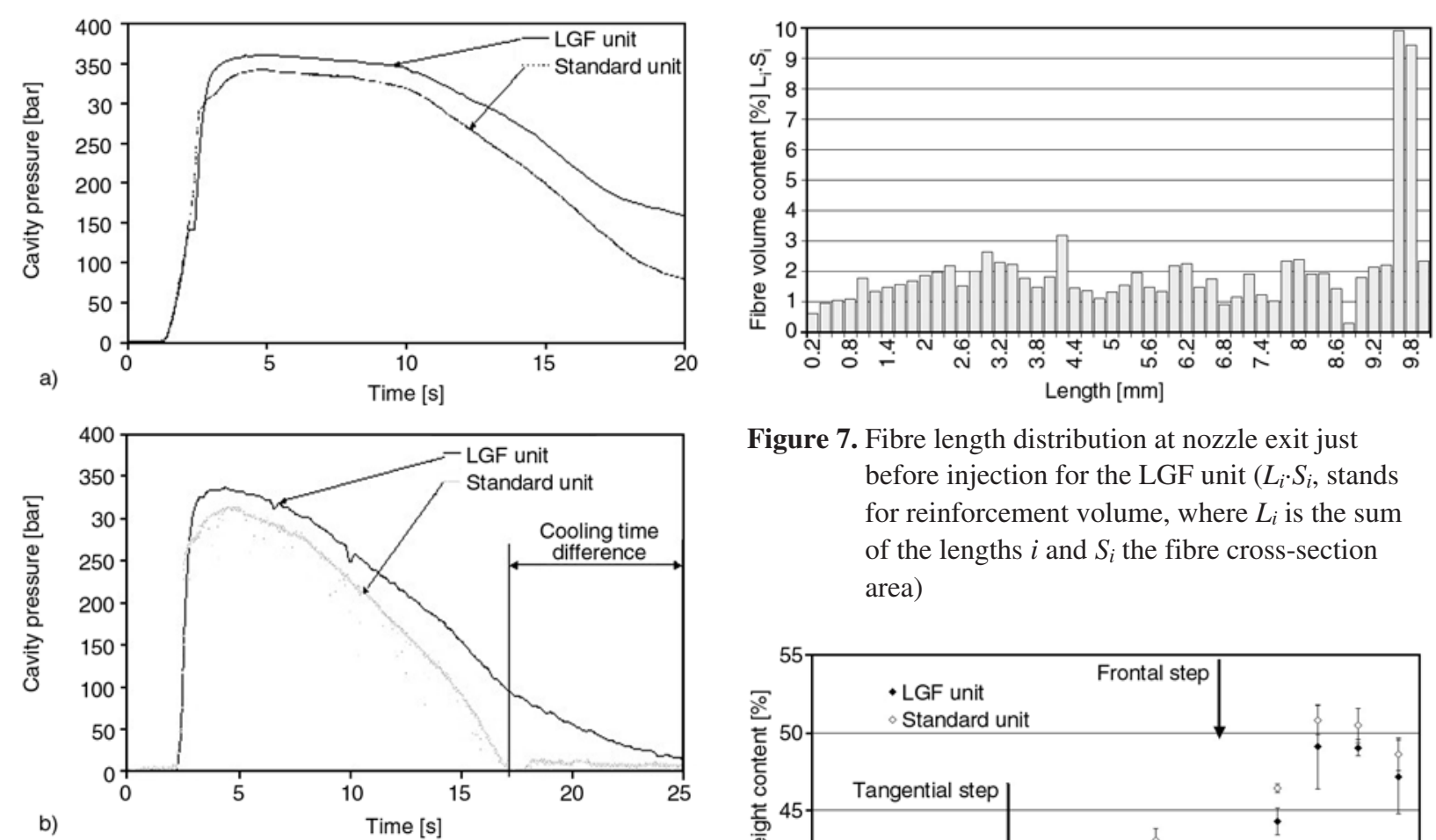

Figure 6. Influence of plasticating unit type on cavity pressure for LGF $40 \mathrm{wt} \%$ - $10 \mathrm{~mm}$ injectionmoulded parts. a) from $40 \mathrm{~mm}$ of the gate, b) from $220 \mathrm{~mm}$ the gate

$80 \%$ at the nozzle exit (Table 4). A bimodal residual fibre length distribution is obtained, which shows that more than $20 \%$ of fibres have a length higher than $5 \mathrm{~mm}$ (Figure 7). However, this significant increase of average residual fibre length does not necessarily allow improving the composite mechanical performances, as previously mentioned (Table 3). This result is logical considering the critical fibre length value $(1.24 \mathrm{~mm}$, calculated in reference [5]), which is already over passed with the standard plasticating unit as soon as the processing conditions are well adapted (low injection speed, high temperatures) to LFT injection moulding.

The fibre content distribution throughout the part is the same for both plasticating units whatever direction is considered (flow (Figure 8) or transverse

Figure 7. Fibre length distribution at nozzle exit just before injection for the LGF unit $\left(L_{i} \cdot S_{i}\right.$, stands for reinforcement volume, where $L_{i}$ is the sum of the lengths $i$ and $S_{i}$ the fibre cross-section area)

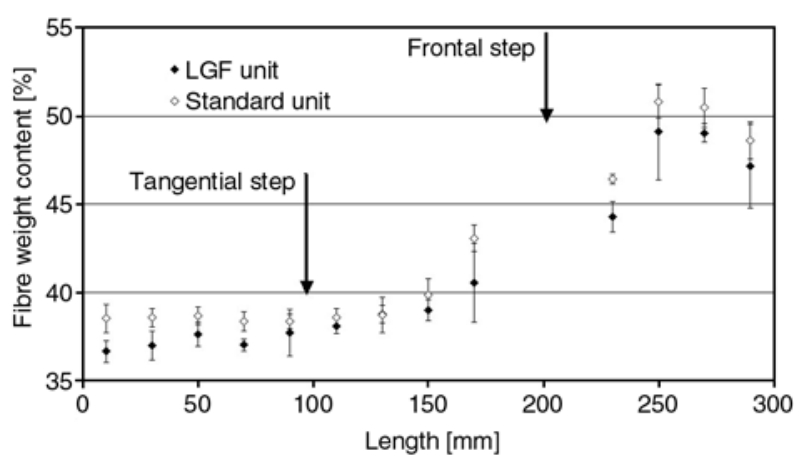

Figure 8. Influence of plasticating unit type on fibre weight content distribution along the flow axis of LGF $40 \mathrm{wt} \%$ - $10 \mathrm{~mm}$ injection-moulded parts

directions). Nevertheless, the increase of the average residual fibre length tends to modify the fibre orientation profile through the thickness, as shown on Figure 9 at the beginning of the part. Whatever the plasticating unit may be it is possible to consider that the composite part shows a quite similar symmetric structure. Two skin layers (one on each side) are noticed. Each skin layer is made of a thin zone just at the cavity wall, where the fibres are randomly distributed due to the fountain flow, followed by a peak showing a much higher orientation

Table 4. Influence of plasticating unit on the residual fibre length of LGF $40 \mathrm{wt} \%-10 \mathrm{~mm}$ injection-moulded parts

\begin{tabular}{|l|c|c|c|c|c|c|}
\hline \multicolumn{1}{|c|}{ Plasticating unit type } & \multicolumn{3}{c|}{ Standard unit } & \multicolumn{3}{c|}{ LGF unit } \\
\hline \multicolumn{1}{|c|}{ Location of the measurement $^{\text {S.o.M.* }}$} & $\begin{array}{c}\text { Beginning } \\
\text { section }\end{array}$ & $\begin{array}{c}\text { End } \\
\text { section }\end{array}$ & S.o.M.* $^{*}$ & $\begin{array}{c}\text { Beginning } \\
\text { section }\end{array}$ & $\begin{array}{c}\text { End } \\
\text { section }\end{array}$ \\
\hline${\text { Number-average length }[\mathrm{mm}]^{\mathrm{a}}}^{\mathrm{a}}$ & 1.64 & 1.50 & 1.80 & 2.97 & 2.89 & 2.89 \\
\hline${\text { Weight-average length }[\mathrm{mm}]^{\mathrm{a}}}^{\text {Standard deviation }[\mathrm{mm}]^{\mathrm{a}}}$ & 4.17 & 3.18 & 3.84 & 5.97 & 4.97 & 5.84 \\
\hline Polydispersity ratio $^{\mathrm{a}}$ & 2.04 & 1.59 & 1.92 & 3.00 & 2.45 & 2.92 \\
\hline Fibre aspect ratio $^{\mathrm{b}}$ & 2.54 & 2.12 & 2.13 & 2.01 & 1.72 & 2.02 \\
\hline
\end{tabular}

aaccording to ISO 22314; bnumber-average fibre length/fibre diameter ratio.

*Shot out of the mould (screw purging). 


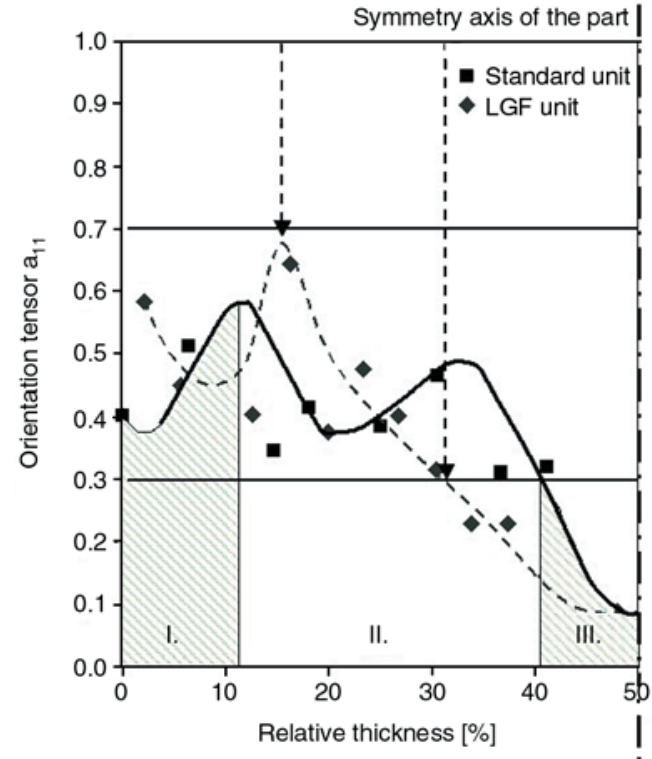

Figure 9. Influence of plasticating unit type on the orientation profile through the thickness at the beginning section of LGF $40 \mathrm{wt} \%$ - $10 \mathrm{~mm}$ injectionmoulded parts $(\mathrm{I}=$ skin layer; $\mathrm{II}=$ intermediate layer; III = core layer)

( $a_{11}$ reaching almost 0.6 or 0.7 ) due to the shear flow influenced by the injection speed and the nonisotherm effects. Then two randomly oriented intermediate zones resulting from the secondary flows during the holding stage are noticed. Finally, a transversely oriented layer, defined as the core layer (where $a_{11}<0.30$ ), is visible in the centre of the parts. The relative thickness of the core layer increases however by $80 \%$ (the ratio of the core to the total thickness increases from 0.2 to 0.36 ) when a LGF plasticating unit is used (Figure 9). The space hindrance effect due to the higher average residual fibre length decreases the fibres mobility and thus their aptitude to orient in the flow direction even if the viscosity of the melt is lower. This evolution of the skin/core ratio may explain the decrease of the longitudinal modulus $E_{1}$ (variation of $\sigma_{1}$ is not significant) and of the anisotropy ratio as well as the slight improvement of the transverse flexural strength $\sigma_{2}$ at this location. The decrease of the transverse modulus $E_{2}$ is more difficult to explain. However one should keep in mind that the layer of higher stiffness (well-aligned fibres in the longitudinal direction of the test coupon) is located in the sample centre in case of a transverse loading, which is the less favourable configuration to increase the sample flexural modulus.

In summary, a LGF dedicated screw and non-return valve design mainly allows limiting the fibre break- age thanks to a limitation of the stresses imposed on fibres during plastication. However, increasing the residual average fibre length above the critical fibre length does not significantly improve the flexural properties of the injection-moulded parts compared to those observed on injection mouldings using a standard plasticating unit as soon as the specific LGF moulding rules are respected in the latter case (e.g. low injection speed, high temperatures). Nevertheless, increasing the fibre aspect ratio (length/ diameter ratio) may contribute to improve other mechanical properties such as the impact resistance in a significant extent, justifying therefore the interest in LGF dedicated plasticating units [17].

\subsection{Initial fibre fraction influence}

The initial fibre length being kept constant and equal to $10 \mathrm{~mm}$, the initial fibre fraction increase from $40 \mathrm{wt} \%$ up to $50 \mathrm{wt} \%$ significantly improves all the flexural properties as expected. In particular the longitudinal flexural strength increases by $22 \%$ and the longitudinal modulus by $30 \%$ at the end of the part (Table 5). The moulded part also tends to be more isotropic in its beginning section (i. e. before the sharp steps inducing flow perturbation). Moreover, the cavity pressure records indicate an average pressure reduction of 30 bars as well as a shorter freezing time in particular at the end of the part $(\Delta t=3 \mathrm{~s})$ when the initial fibre fraction is increased up to $50 \mathrm{wt} \%$. In that case, the thermomechanical behaviour appears to be close to the one previously observed for the LGF $40 \mathrm{wt} \%$ formulation moulded with the standard plasticating unit.

At the nozzle exit just before injection, the LGF $50 \mathrm{wt} \%$ formulation keeps about one half of its initial fibre length (e.g. fibre breakage amount limited to $57 \%$ ). The high weight-average fibre length value also indicates that there is an important

Table 5. Influence of initial fibre fraction on flexural properties of injection-moulded parts (LGF unit)

\begin{tabular}{|c|c|c|}
\hline \multirow[t]{2}{*}{ Property $^{*}$} & \multicolumn{2}{|c|}{$\begin{array}{c}\text { Property variation for LGF } \\
50 \mathrm{wt} \%-10 \mathrm{~mm} \text { (compared } \\
\text { to LGF } 40 \mathrm{wt} \%-10 \mathrm{~mm} \text { ) }\end{array}$} \\
\hline & $\begin{array}{c}\text { Beginning } \\
\text { section }\end{array}$ & $\begin{array}{c}\text { End } \\
\text { section }\end{array}$ \\
\hline Longitudinal strength $\sigma_{1}[\mathrm{MPa}]$ & $+9 \%$ & $+22 \%$ \\
\hline Transverse strength $\sigma_{2}[\mathrm{MPa}]$ & $+15 \%$ & $+17 \%$ \\
\hline Longitudinal modulus $E_{1}[\mathrm{MPa}]$ & $+16 \%$ & $+30 \%$ \\
\hline Transverse modulus $E_{2}[\mathrm{MPa}]$ & $+6 \%$ & $+3 \%$ \\
\hline Anisotropy ratio $\sigma_{1} / \sigma_{2}$ & $-5 \%$ & $+5 \%$ \\
\hline
\end{tabular}

*Sample location according to Figure 2. 
Table 6. Residual fibre length of LGF $50 \mathrm{wt} \%-10 \mathrm{~mm}$ injection-moulded parts (LGF unit)

\begin{tabular}{|l|c|c|c|}
\hline Location of the measurement & S.o.M.* & $\begin{array}{c}\text { Beginning } \\
\text { section }\end{array}$ & $\begin{array}{c}\text { End } \\
\text { section }\end{array}$ \\
\hline${\text { Number-average length }[\mathrm{mm}]^{\mathrm{a}}}^{\mathrm{a}}$ & 4.22 & 1.63 & 1.69 \\
\hline${\text { Weight-average length }[\mathrm{mm}]^{\mathrm{a}}}^{\mathrm{a}}$ & 7.22 & 4.46 & 3.94 \\
\hline${\text { Standard deviation }[\mathrm{mm}]^{\mathrm{a}}}$ & 3.58 & 2.15 & 1.95 \\
\hline Polydispersity ratio $^{\mathrm{a}}$ & 1.71 & 2.73 & 2.32 \\
\hline Fibre aspect ratio $^{\mathrm{b}}$ & 281 & 108 & 113 \\
\hline
\end{tabular}

aaccording to ISO 22314;

bnumber-average fibre length/fibre diameter ratio.

*Shot out of the mould (screw purging).

amount of long fibres in the distribution (Table 6). Decreasing the matrix weight fraction within the pellets tends to diminish the melt viscosity in the barrel more rapidly and thus to reduce the stresses imposed to the fibres. This trend may be explained by the fact that the thermal conductivity of glass is better than that of the polymer matrix. Thermal exchanges in the thin matrix channel in the barrel occur more rapidly and the material therefore reaches its melting point earlier. In the injectionmoulded part however, the amount of fibre breakage is much higher because of the fibre/fibre interactions, which are logically higher at higher initial fibre fractions. Nevertheless the fibre aspect ratio remains higher than 100 and the average fibre length still overpasses the critical fibre length.

The orientation profile through the thickness is also comparable to the one noticed for the LGF $40 \mathrm{wt} \%$ formulation moulded with the standard plasticating unit (Figure 9). The orientation level of the skin layers (peak at $a_{11}>0.7$ ) tends however to be slightly higher. Moreover, the core layer is thinner when the initial fibre fraction increases (Figure 10). The lower residual fibre length actually increases their mobility and their aptitude to orient.

The fibre content distribution along the flow axis is almost the same whatever the initial fibre fraction may be (Figure 11). However, the perturbations induced by the geometrical discontinuities are less important at higher fibre fractions due to the presence of shorter fibre in the melt. In the width direction, the fibre content difference between the edges and the middle axis of the injection-moulded parts does not exceed $6 \mathrm{wt} \%$ for the LGF $50 \mathrm{wt} \%$ reference (for instance at the end section) whereas it reaches more than $8 \mathrm{wt} \%$ for the LGF $40 \mathrm{wt} \%$ formulation. As a consequence the in-plane homogeneity of the part is slightly better for higher initial fibre fractions. Regarding the fibre distribution

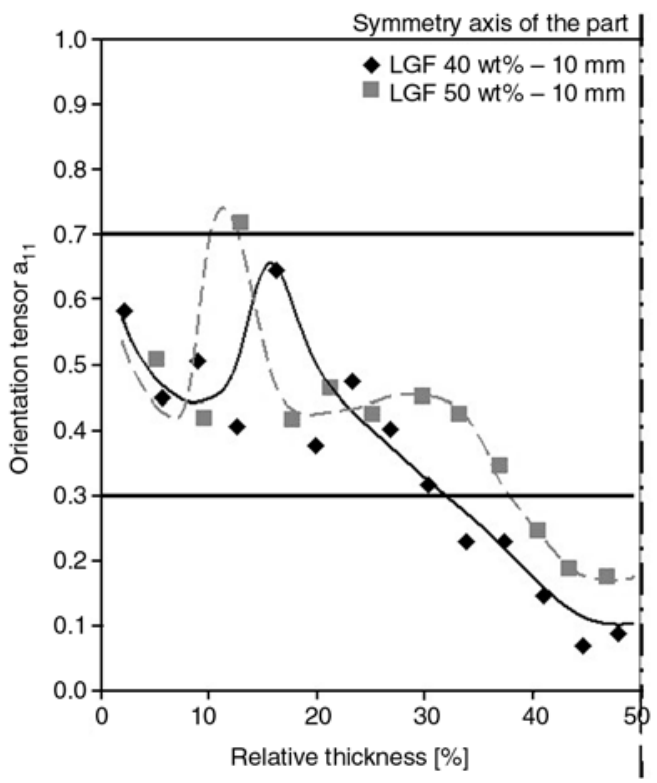

Figure 10. Influence of initial fibre fraction on fibre orientation through the thickness at the beginning section of injection-moulded parts (LGF unit, $10 \mathrm{~mm}$ initial fibre length)

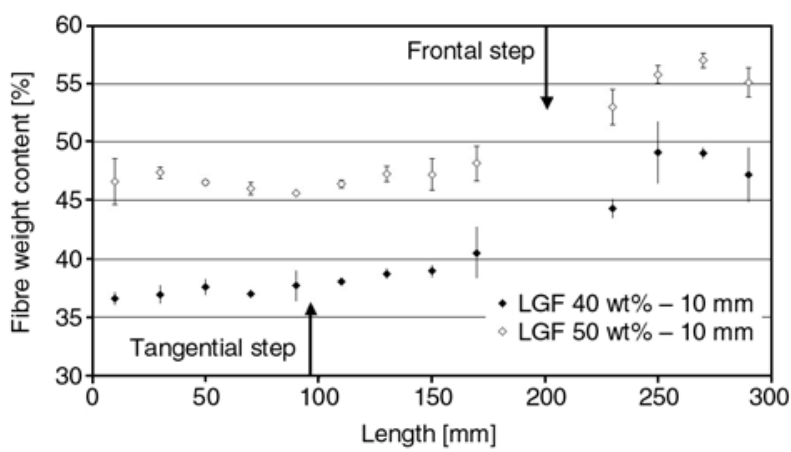

Figure 11. Influence of initial fibre content on fibre weight content distribution along the flow axis of the injection-moulded part (LGF unit, $10 \mathrm{~mm}$ initial fibre length)

through the thickness, the fibre content is lower in the skin layers and higher in the core whatever the initial fibre weight fraction may be. This segregation effect is visible for example in the beginning section of the part (Figure 12). These results confirm the observations made by other authors [15, $16,17]$ on different long fibre reinforced thermoplastics injection-mouldings, such a fibre segregation being attributed to fibre breakage or pull-out at the interface between frozen and molten layers and subsequent sweeping along during the filling stage of the injection-moulding cycle.

In summary, increasing the initial fibre fraction in the LFT pellets allows to improve the flexural mechanical properties due the globally higher fibre content. The average residual fibre length in the 


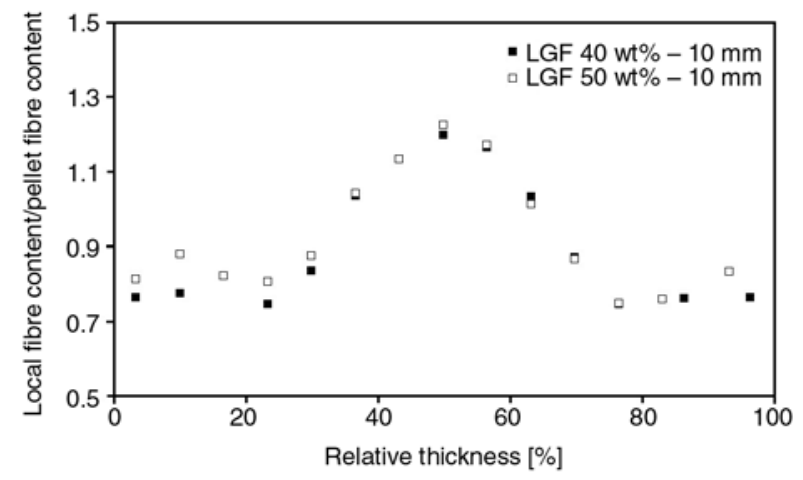

Figure 12. Influence of initial fibre content on fibre distribution through the thickness at $50 \mathrm{~mm}$ from the gate of the injection moulded part (LGF unit, $10 \mathrm{~mm}$ initial fibre length)

part decreases however as there are more fibre/fibre interactions. As a consequence, the fibre orientation profile through the part thickness is slightly modified. The skin layers are a bit more oriented and the transversally oriented core layer is thinner due to the better mobility of shorter fibres. The concentration gradients in the injection-moulded parts do not depend a lot on the initial fibre fraction.

\subsection{Initial fibre length influence}

The initial fibre fraction in the pellets being kept constant and equal to $50 \mathrm{wt} \%$, the increase of the initial fibre length from 10 to $25 \mathrm{~mm}$ globally decreases the flexural properties and increases the anisotropy ratio (Table 7).

At the nozzle exit just before injection, the weightaverage residual fibre length of the LGF $25 \mathrm{~mm}$ pellets is multiplied by 1.5 compared to the LGF $10 \mathrm{~mm}$ pellets, whereas the number-average fibre length does not change (Table 8 ). This means that the amount of long fibres is high. $15 \%$ of the fibres are actually longer than $10 \mathrm{~mm}$. This result confirms the efficiency of the LGF dedicated plasticating unit and the proper choice of the injectionmoulding conditions. However, the fibre breakage amount within the part is then very high in particular in the end section, even though the residual number- and weight-average fibre lengths remain higher than those of the LGF $10 \mathrm{~mm}$ pellets (Table 6). Considering the prejudicial effect on the flexural mechanical properties, there is obviously no benefit to increase the initial fibre length too much to obtain better residual mechanical performances in the case of the standard injection moulding process.
Table 7. Influence of initial fibre length influence on flexural properties of injection-moulded parts (LGF unit)

\begin{tabular}{|l|c|c|}
\hline \multirow{2}{*}{ Property* } & \multicolumn{2}{|c|}{$\begin{array}{c}\text { Property variation for LGF } \\
\text { 50 wt } \%-25 ~ \mathbf{~ m m} \text { (compared } \\
\text { to LGF 50 wt } \% \text { - 10 } \mathbf{~ m m})\end{array}$} \\
\cline { 2 - 3 } & $\begin{array}{c}\text { Beginning } \\
\text { section }\end{array}$ & $\begin{array}{c}\text { End } \\
\text { section }\end{array}$ \\
\hline Longitudinal strength $\sigma_{1}[\mathrm{MPa}]$ & $\approx$ & $-8 \%$ \\
\hline Transverse strength $\sigma_{2}[\mathrm{MPa}]$ & $-14 \%$ & $-35 \%$ \\
\hline Longitudinal modulus $E_{1}[\mathrm{MPa}]$ & $\approx$ & $-6 \%$ \\
\hline Transverse modulus $E_{2}[\mathrm{MPa}]$ & $\approx$ & $\approx$ \\
\hline Anisotropy ratio $\sigma_{1} / \sigma_{2}$ & $+11 \%$ & $+40 \%$ \\
\hline
\end{tabular}

*Sample location according to Figure 2.

Table 8. Residual fibre length of LGF $50 \mathrm{wt} \%-25 \mathrm{~mm}$ injection-moulded parts (LGF unit)

\begin{tabular}{|l|c|c|c|}
\hline Location of the measurement & S.o.M.* & $\begin{array}{c}\text { Beginning } \\
\text { section }\end{array}$ & $\begin{array}{c}\text { End } \\
\text { section }\end{array}$ \\
\hline Number-average length $[\mathrm{mm}]^{\mathrm{a}}$ & 4.37 & 2.40 & 1.85 \\
\hline Weight-average length [mm] $^{\mathrm{a}}$ & 11.11 & 5.19 & 3.88 \\
\hline${\text { Standard deviation }[\mathrm{mm}]^{\mathrm{a}}}^{\mathrm{a}}$ & 5.47 & 2.59 & 1.94 \\
\hline Polydispersity ratio $^{\mathrm{a}}$ & 2.54 & 2.16 & 2.09 \\
\hline Fibre aspect ratio $^{\mathrm{b}}$ & 291 & 160 & 123 \\
\hline
\end{tabular}

aaccording to ISO 22314;

bnumber-average fibre length/fibre diameter ratio.

*Shot out of the mould (screw purging).

The fibre content distribution along the flow axis clearly shows a fibre accumulation near the gate and a huge data scattering, which reveals the existence of fibre clusters (Figure 13). The segregation effect induced by the sharp geometrical discontinuities is also amplified. In the width direction, the fibre accumulation also appears near the gate, however only on the middle axis of the part (Figure 14). As the feeding of the fan gate occurs by means of a circular channel (Figure 1), the extensional radial stresses induced in the divergent part of the fan gate

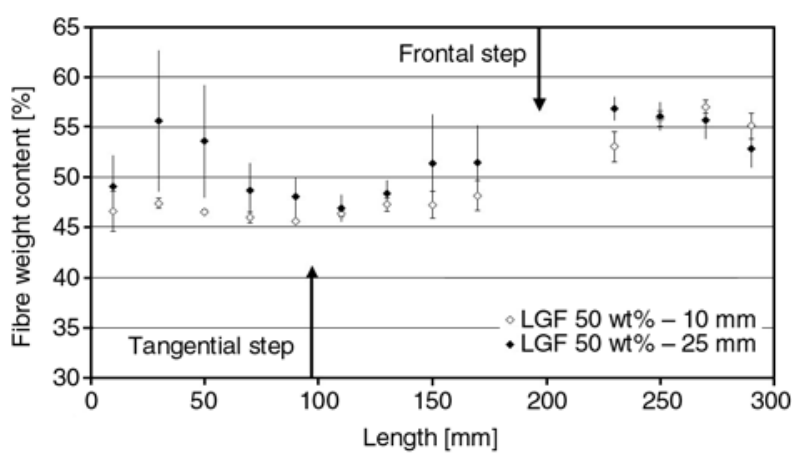

Figure 13. Influence of initial fibre length on fibre weight content distribution along the flow axis of injection-moulded parts (LGF unit, $50 \mathrm{wt} \%$ initial fibre content) 


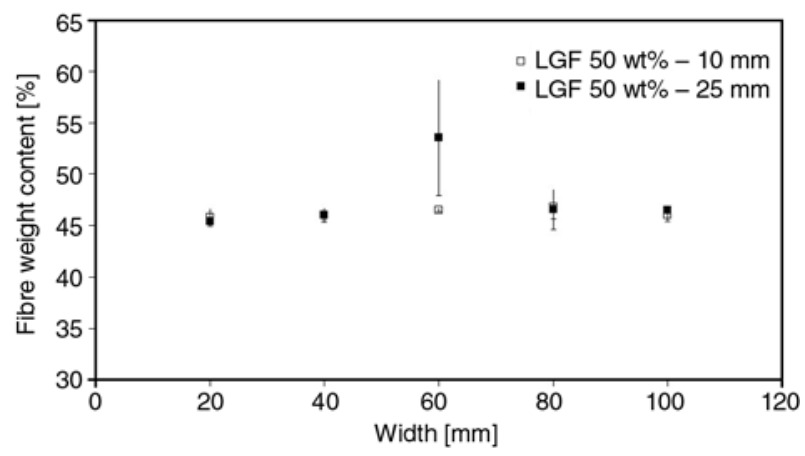

Figure 14. Influence of initial fibre length on fibre weight content distribution along the width at $50 \mathrm{~mm}$ from the gate of the injection-moulded part (LGF unit, $50 \mathrm{wt} \%$ initial fibre content)

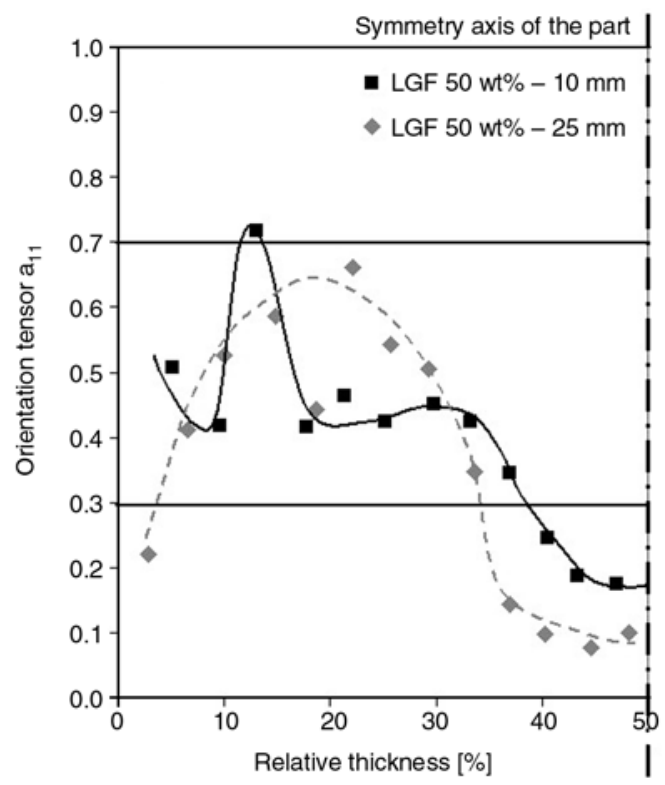

Figure 15. Influence of initial fibre length on fibre orientations through the thickness at $50 \mathrm{~mm}$ from the gate of injection-moulded parts (LGF unit, $50 \mathrm{wt} \%$ initial fibre content)

appear to be not high enough to warrant good fibre dispersion. These results also indicate an instability of the moulding process when very long fibre reinforced thermoplastic pellets are used. This instability is confirmed by the high scatter in metering times observed during the plastication stage.

The fibre orientation through the thickness is largely modified by the high average residual length. In the beginning section for example (Figure 15), the composite structure is characterized by the absence of high orientation peaks and is made of two randomly oriented skin layers and one perpendicularly orientated core layer, each layer representing almost one third of the whole part thickness. The strong interactions between fibres and with the surrounding molten polymer or the mould

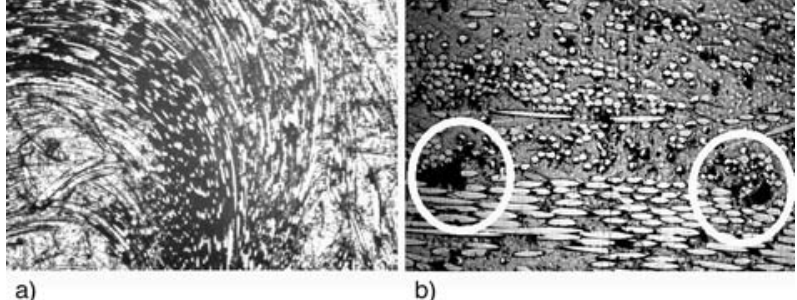

b)

Figure 16. Fibre cluster a) and local porosity b) at the beginning location of injection-moulded parts (LGF unit, $50 \mathrm{wt} \%$ initial fibre content)

have decreased the fibre mobility and increased the fibre breakage and the structural heterogeneities.

The decrease of flexural properties may be explained by the existence of numerous fibre clusters in the part and an increase of the local void content (Figure 16). Although the void content is mainly influenced by the holding pressure, it is not independent from the fibre content and length. Increasing the fibre content and the fibre length involves a higher viscosity. This means a lower compaction efficiency at a given holding pressure level. Moreover, the thermal conductivity of glass is better than that of the polymer matrix; the cooling speed is therefore higher and the efficiency of the holding stage consequently lower (the time during which the holding pressure is applied on the polymer is shorter).

In summary, increasing the initial fibre length of the LFT pellets too much does not warrant better flexural properties of injection-moulded parts. Fibre clustering and local porosity are signs of an instability of the injection-moulding process. In that case, the use of other processes such as extrusion-compression moulding or injection-compression moulding would be recommended to further improve the mechanical properties.

\section{Conclusions}

This work has allowed to define guidelines to improve the mechanical performances of PA 6-6/ glass LFT injection mouldings. Different solutions have been considered:

- Using a LFT dedicated plasticating unit (lower compression ratio, longer compression section, higher flight depth in feed section, shorter sliding ring length) has decreased the fibre breakage amount by about $80 \%$ at the nozzle exit. However, increasing the residual average fibre length above the critical fibre length does not signifi- 
cantly improve the flexural properties. Standard plasticating units may be used as soon as the injection moulding conditions are adapted to LFT processing (e.g. low injection speed, high temperatures).

- Increasing the initial fibre content in the LFT pellets (from 40 to $50 \mathrm{wt} \%$ ) has logically permitted to improve the flexural properties.

- Increasing the initial fibre length (from 10 to $25 \mathrm{~mm}$ ) has not brought any improvement of the properties and has even amplified the structural heterogeneities (fibre clusters and local porosity) and the anisotropy of the parts.

\section{Acknowledgments}

Thanks are due to Ticona Gmbh for supplying LFT raw materials and to European Union (European Funds for Regional Development 'FEDER') for contributing to funding the equipment.

\section{References}

[1] Yilmazer U., Cansever M.: Effects of processing conditions on the fiber length distribution and mechanical properties of glass fiber reinforced Nylon-6. Polymer Composites, 23, 61-71 (2002).

[2] Obieglo G., Roller B.: Injection moulding of glass fibre reinforced polyamides. Influence of processing conditions on glass fibre distribution and the properties of the mouldings. Kunststoffe, 75, 179-181 (1985).

[3] Metten M., Cremer M.: Injection moulding of longfibre-reinforced thermoplastics. Kunststoffe, 90, 8083 (2000).

[4] Hafellner R., Picher M., Wörndle R.: Injection moulding long fibres. Kunststoffe, 90, 44-48 (2000).

[5] Lafranche E., Krawczak P., Ciolczyk J. P., Maugey P.: Injection moulding of long glass fiber reinforced polyamide 66: Processing condition/microstructure/ flexural properties relationship. Advances in Polymer Technology, 24, 114-131 (2005).

[6] Gérard P.: Maîtrise et optimisation de l'anisotropie et de l'hétérogénéité des composites a matrices thermoplastiques renforcés de fibres de verre courtes en injection (Control and optimisation of anisotropy and heterogeneity of injection-moulded short glass fibre reinforced thermoplastic composites). PhD Thesis, University of Lille I - Ecole des Mines de Douai (1998).

[7] Bijsterbosch H., Gaymans R. J.: Polyamide 6-long glass fiber injection moldings. Polymer Composites, 16, 363-369 (1995).

[8]. Thomason J. L.: The influence of fibre properties on the properties of glass-fibre-reinforced polyamide 66 . Journal of Composite Materials, 34, 158-172 (2000).
[9] Ranganathan S., Advani S. G.: Characterization of orientation clustering in short-fiber composites. Journal of Polymer Science: Part B, Polymer Physic, 28, 2651-2672 (1990).

[10] Karger-Kocsis J., Harmia T., Czigany T.: Comparison of the fracture and failure behavior of polypropylene composites reinforced by long glass fibers and glass mats. Composite Science and Technology, 54, 287298 (1995).

[11] Thomason J. L., Vlug M. A.: Influence of fibre length and concentration on the properties of glass fibre-reinforced polypropylene: 4. Impact properties. Composites: Part A, 28, 277-288 (1997).

[12] Bay R. S., Tucker C. L.: Stereological measurement and error estimates for three-dimensional fiber orientation. Polymer Engineering and Science, 32, 240-253 (1992).

[13] Gupta M., Wang K. K.: Fiber orientation and mechanical properties of short-fiber reinforced injectionmolded composites: simulated and experimental results. Polymer Composites, 14, 367-382 (1993).

[14] Stokes V. K., Inzinna L. P., Liang E. W., Trantina G. C., Woods J. T.: A phenomenological study of the mechanical properties of long fiber filled injection molded thermoplastic composites. Polymer Composites, 21, 696-710 (2000).

[15] O'Regan D., Akay M.: The distribution of fibre lengths in injection moulded polyamide composite components. Journal of Materials Processing Technology, 56, 282-291 (1996).

[16] Toll S., Anderson P.O.: Microstructure of long and short fiber reinforced injection molded polyamide. Polymer Composites, 14, 116-125 (1993).

[17] Cilleruelo L.: Analyse des relations mise en ouvre/structure/propriétés mécaniques du PET renforcé de fibres de verre longues (Analysis of the processing/structure/mechanical properties relationship of long glass fibre reinforced PET). PhD Thesis, University of Lille I - Ecole des Mines de Douai (2005).

[18] Kamal M. R., Singh P.: The distribution of fiber phase properties in injection molded short glass fiber composites. in 'SPE ANTEC 89 Technical Papers, San Diego, USA' 240 (1989).

[19] Advani S. G., Tucker C. L.: The use of tensors to describe and predict fibre orientation in short fibre composites. Journal of Rheology, 31, 751-784 (1987).

[20] Ticona Celstran-Compel Fiberod: Long fibre reinforced thermoplastics (LFT). Technical data sheet, Ticona Gmbh (1999).

[21] Coulon A., Analyse des relations entre conditions de transformation et comportement mécanique de pieces moulées par injection en polyamide renforcé de fibres de verre longues (Analysis of the relationship between processing conditions and mechanical behaviour of injection-moulded long glass fibre reinforced polyamide parts). PhD Thesis, University of Lille I - Ecole des Mines de Douai (2007). 